\title{
SINGULAR SUPPORTS FOR CHARACTER SHEAVES ON A GROUP COMPACTIFICATION
}

\author{
Xuhua He and George Lusztig
}

\begin{abstract}
Let $G$ be a semisimple adjoint group over $\mathbf{C}$ and $\bar{G}$ be the De ConciniProcesi completion of $G$. In this paper, we define a Lagrangian subvariety $\Lambda$ of the cotangent bundle of $\bar{G}$ such that the singular support of any character sheaf on $\bar{G}$ is contained in $\Lambda$.
\end{abstract}

1.1. In this paper all algebraic varieties are assumed to be over a fixed algebraically closed field of characteristic 0 .

If $X$ is a smooth variety, let $T^{*} X$ be the cotangent bundle of $X$. For any morphism $\alpha: X \rightarrow Y$ of smooth varieties and $x \in X$, we write $\alpha^{*}: T_{\alpha(x)}^{*} Y \rightarrow T_{x}^{*} X$ for the map induced by $\alpha$. If moreover, $\alpha: X \rightarrow Y$ is a locally trivial fibration with smooth connected fibres and $\Lambda$ is a closed Lagrangian subvariety of $T^{*} Y$, then let $\alpha^{\star}(\Lambda)=\cup_{x \in X} \alpha^{*}\left(\Lambda \cap T_{\alpha(x)}^{*} Y\right) \subset T^{*} X$. Then

(a) $\alpha^{\star}(\Lambda)$ is a closed Lagrangian subvariety of $T^{*} X$. Moreover, the set of irreducible components of $\Lambda$ is naturally in bijection with the set of irreducible components of $\alpha^{\star}(\Lambda)$.

Let $X, Y$ be smooth irreducible varieties and let $\alpha: X \rightarrow Y$ be a principal $P$-bundle for a free action of a connected linear algebraic group $P$ on $X$.

(b) If $\Lambda^{\prime}$ is a closed Lagrangian subvariety of $T^{*} X$ stable under the P-action then $\Lambda^{\prime}=\alpha^{\star}(\Lambda)$ for a unique closed Lagrangian subvariety $\Lambda$ of $T^{*} Y$.

Let $X$ be a smooth irreducible variety and let $i: Y \rightarrow X$ be the inclusion of a locally closed smooth irreducible subvariety. Let $\Lambda$ be a closed Lagrangian subvariety of $T^{*} Y$. Let $i_{\star}(\Lambda)$ be the subset of $T^{*} X$ consisting of all $\xi \in T_{x}^{*} X$ such that $x \in Y$ and the image of $\xi$ under the obvious surjective map $T_{x}^{*} X \rightarrow T_{x}^{*} Y$ belongs to $\Lambda \cap T_{x}^{*} Y$. Note that

(c) $i_{\star}(\Lambda)$ is a locally closed Lagrangian subvariety of $T^{*} X$. Moreover, the set of irreducible components of $i_{\star}(\Lambda)$ is naturally in bijection with the set of irreducible components of $\Lambda$.

2000 Mathematics Subject Classification. 20 G99.

X. H. is is supported by NSF grant DMS-0111298. G.L. is supported in part by NSF grant DMS-0243345.

Typeset by $\mathcal{A} \mathcal{M} \mathcal{S}-\mathrm{T}_{\mathrm{E}} \mathrm{X}$ 
For an algebraic variety $X$ we write $\mathcal{D}(X)$ for the bounded derived category of constructible $\overline{\mathbf{Q}}_{l}$-sheaves on $X$ where $l$ is a fixed prime number. For $X$ smooth and $C \in \mathcal{D}(X)$, we denote by $S S(X)$ the singular support of $C$ (a closed Lagrangian subvariety of $\left.T^{*} X\right)$. Let $A$ be a connected linear algebraic group acting on a smooth variety $X$ and let $B$ be a connected subgroup of $A$. Let $\mu_{A}: T^{*} X \rightarrow$ Lie $(A)^{*}$ be the moment map of the $A$-action on $X$. Consider the diagram $X \stackrel{p r_{1}}{\longleftarrow}$ $A \times X \stackrel{p r_{2}}{\longrightarrow} A \times_{B} X \stackrel{\pi}{\rightarrow} X$ where $B$ acts on $A \times X$ by $b:(a, x) \mapsto\left(a b^{-1}, b x\right)$, $A \times_{B} X$ is the quotient space and $\pi(a, x)=a x$. Then for any $B$-equivariant perverse sheaf $C$ on $X$ there is a well defined perverse sheaf $C^{\prime}$ on $A \times_{B} X$ such that $p r_{2}^{*} C^{\prime}=p r_{1}^{*} C$ up to a shift. We set $\Gamma_{B}^{A}(C)=\pi_{*} C^{\prime} \in \mathcal{D}(X)$. By $[\mathrm{MV}, 1.2]$ we have

(d) $S S\left(\Gamma_{B}^{A}(C)\right) \subset \overline{A \cdot S S(C)}$.

On the other hand, we have

(e) $S S(C) \subset \mu_{B}^{-1}(0)$.

Indeed, if $p_{1}: B \times X \rightarrow X$ is the action and $p_{2}: B \times X \rightarrow X$ is the second projection we have $p_{1}^{*}(C)=p_{2}^{*}(C)$. Hence $S S\left(p_{1}^{*}(C)\right)=S S\left(p_{2}^{*}(C)\right)$. Using $[\mathrm{KS}$, 4.1.2] we can rewrite this as $p_{1}^{\star}(S S(C))=p_{2}^{\star}(S S(C))$. Hence if $x \in X$ and $\xi \in T_{x}^{*} X \cap S S(C)$ then the image of $\xi$ under the map $T_{x}^{*} X \rightarrow T_{1}^{*}(B)$ induced by $B \rightarrow X, b \mapsto b x$ is 0 . This proves (e).

1.2. Let $G$ be a connected reductive algebraic group. Let $\mathfrak{g}=\operatorname{Lie}(G)$. Let $\mathcal{N}$ be the variety of nilpotent elements in $\mathfrak{g}^{*}$. Let $B$ be a Borel subgroup of $G$. Let $K$ be a closed connected subgroup of $G$. Then $B_{K}=B \cap K$ is a parabolic subgroup of $K$. Assume that $G$ acts on a smooth variety $X$. Let $C$ be a $B_{K}$-equivariant perverse sheaf on $X$; assume also that there exists a finite covering $a: \tilde{B} \rightarrow B$ such that $C$ is $\tilde{B}$-equivariant for the $\tilde{B}$-action $\tilde{b}: x \mapsto a(\tilde{b}) x$ on $X$. By $1.1(\mathrm{e})$ we have $\mu_{\tilde{B}}(S S(C))=0$. Since $\operatorname{Lie}(\tilde{B})=\operatorname{Lie}(B)$ we then have $\mu_{B}(S S(C))=0$. It follows that $\mu_{G}(S S(C))$ is contained in the kernel of the obvious map $\mathfrak{g}^{*} \rightarrow \operatorname{Lie}(B)^{*}$ hence is contained in $\mathcal{N}$. Since $\mathcal{N}$ is stable under the coadjoint action we have $\mu_{G}(K \cdot S S(C))=K \mu_{G}(S S(C)) \subset \mathcal{N}$. Using this together with $1.1(\mathrm{~d})$ and the fact that $\mu_{G}^{-1}(\mathcal{N})$ is closed in $T^{*} X$ we see that $S S\left(\Gamma_{B_{K}}^{K}(C)\right) \subset \mu_{G}^{-1}(\mathcal{N})$. Applying 1.1(e) to $\Gamma_{B_{K}}^{K}(C), K$ instead of $C, B$ we see that $S S\left(\Gamma_{B_{K}}^{K}(C)\right) \subset \mu_{K}^{-1}(0)=\mu_{G}^{-1}\left(\operatorname{Lie}(K)^{\perp}\right)$ where $\operatorname{Lie}(K)^{\perp} \subset \mathfrak{g}^{*}$ is the annihilator of $\operatorname{Lie}(K) \subset \mathfrak{g}$. Thus we have

$$
S S\left(\Gamma_{B_{K}}^{K}(C)\right) \subset \mu_{G}^{-1}\left(\operatorname{Lie}(K)^{\perp} \cap \mathcal{N}\right) .
$$

1.3. We now replace $G, \mathfrak{g}, B, K, B_{K}, X, C$ by $G \times G, \mathfrak{g} \times \mathfrak{g}, B \times B, G_{\Delta}, B_{\Delta}, X^{\prime}, C^{\prime}$ where $G_{\Delta}=\left\{\left(g, g^{\prime}\right) \in G \times G ; g=g^{\prime}\right\}, B_{\Delta}=\left\{\left(g, g^{\prime}\right) \in B \times B ; g=g^{\prime}\right\}, X^{\prime}$ is a smooth variety with a given action of $G \times G$ and $C^{\prime}$ is a $B_{\Delta}$-equivariant perverse sheaf on $X^{\prime}$; we assume that there exists a finite covering $a^{\prime}: \tilde{B}^{\prime} \rightarrow B \times B$ such that $C^{\prime}$ is $\tilde{B}^{\prime}$-equivariant for the $\tilde{B}^{\prime}$-action $\tilde{b}^{\prime}: x^{\prime} \mapsto a^{\prime}\left(\tilde{b}^{\prime}\right) x^{\prime}$ on $X^{\prime}$. We have the following special case of $1.2(\mathrm{a})$ :

$$
S S\left(\Gamma_{B_{\Delta}}^{G_{\Delta}}\left(C^{\prime}\right)\right) \subset \mu_{G \times G}^{-1}\left(\mathcal{N}^{-}\right)
$$


where

$$
\mathcal{N}^{-}=\left\{\left(f, f^{\prime}\right) \in \mathfrak{g}^{*} \times \mathfrak{g}^{*} ; f+f^{\prime}=0, f, f^{\prime} \text { nilpotent }\right\} .
$$

1.4. Let $\mathbf{W}$ be the Weyl group of $G$ and let $\mathbf{I}$ be the set of simple reflections in W. Let $G^{\prime}$ be a possibly disconnected algebraic group with identity component $G$ and with a given connected component $D$. Now $G \times G$ acts transitively on $D$ by $\left(g_{1}, g_{2}\right): g \mapsto g_{1} g g_{2}^{-1}$. Hence the moment map $\mu_{G \times G}: T^{*} D \rightarrow \mathfrak{g}^{*} \times \mathfrak{g}^{*}$ is well defined. In $[\mathrm{L} 1,4.5]$ a class of perverse sheaves (called character sheaves) on $D$ is introduced. These appear as constituents of some perverse cohomology sheaf of $\Gamma_{B_{\Delta}}^{G_{\Delta}}\left(C^{\prime}\right)$ for some $C^{\prime}$ as in 1.3 (with $X^{\prime}=D$ ). Hence from 1.3(a) we deduce:

(a) If $K$ is a parabolic character sheaf on $D$ then $S S(K) \subset \mu_{G \times G}^{-1}\left(\mathcal{N}^{-}\right)$.

In the case where $G^{\prime}=G=D$ a statement close to (a) appears in [MV, 2.8] (where it is attributed to the second author) and in [Gi].

1.5. We preserve the setup of 1.4. For any $J \subset \mathbf{I}$ let $\mathcal{P}_{J}$ be the set of parabolic subgroups of $G$ of type $J$. In particular $\mathcal{P}_{\emptyset}$ is the set of Borel subgroups of $G$. For $J \subset \mathbf{I}$ let $\mathbf{W}_{J}$ be the subgroup of $\mathbf{W}$ generated by $J$; let $\mathbf{W}^{J}$ (resp. ${ }^{J} \mathbf{W}$ ) be the set of all $w \in \mathbf{W}$ such that $w$ has minimal length among the elements in $\mathbf{W}_{J} w$ (resp. $w \mathbf{W}_{J}$ ). Let $\delta: \mathbf{W} \stackrel{\sim}{\longrightarrow} \mathbf{W}$ be the isomorphism such that $\delta(\mathbf{I})=\mathbf{I}$ and such that $J \subset \mathbf{I}, P \in \mathcal{P}_{J}, g \in D \Longrightarrow g P g^{-1} \in \mathcal{P}_{\delta(J)}$. Following [L2, 8.18], for $J, J^{\prime} \subset \mathbf{I}$ and $y \in{ }^{J^{\prime}} \mathbf{W} \cap \mathbf{W}^{J}$ such that $\operatorname{Ad}(y)(\delta(J))=J^{\prime}$ we set

$$
Z_{J, y, \delta}=\left\{\left(P, P^{\prime}, g U_{P}\right) ; P \in \mathcal{P}_{J}, P^{\prime} \in \mathcal{P}_{J^{\prime}}, g \in D, \operatorname{pos}\left(P^{\prime}, g P g^{-1}\right)=y\right\}
$$

Now $G \times G$ acts (transitively) on $Z_{J, y, \delta}$ by

$$
\left(g_{1}, g_{2}\right):\left(P, P^{\prime}, g U_{P}\right) \mapsto\left(g_{2} P g_{2}^{-1}, g_{1} P^{\prime} g_{1}^{-1}, g_{1} g g_{2}^{-1}\right)
$$

Hence the moment map $\mu_{G \times G}: T^{*} Z_{J, y, \delta} \rightarrow \mathfrak{g}^{*} \times \mathfrak{g}^{*}$ is well defined. In [L2, Section 11] a class of perverse sheaves (called parabolic character sheaves) on $Z_{J, y, \delta}$ is introduced. These appear as constituents of some perverse cohomology sheaf of $\Gamma_{B_{\Delta}}^{G_{\Delta}}\left(C^{\prime}\right)$ for some $C^{\prime}$ as in 1.3 (with $X^{\prime}=Z_{J, y, \delta}$ ). Hence from 1.3(a) we deduce:

(a) If $K$ is a parabolic character sheaf on $Z_{J, y, \delta}$ then $S S(K) \subset \mu_{G \times G}^{-1}\left(\mathcal{N}^{-}\right)$.

When $J=\mathbf{I}$, this reduces to $1.4(\mathrm{a})$.

1.6. Assume that $G$ is adjoint. Let $\bar{G}$ be the De Concini-Procesi compactification of $G$. Then $G \times G$ acts naturally on $\bar{G}$ extending continuously the action $\left(g_{1}, g_{2}\right)$ : $g \mapsto g_{1} g g_{2}^{-1}$ of $G \times G$ on $G$. Hence the moment map $\mu_{G \times G}: T^{*} \bar{G} \rightarrow \mathfrak{g}^{*} \times \mathfrak{g}^{*}$ is well defined. In [L2] a class of perverse sheaves (called parabolic character sheaves) on $\bar{G}$ is introduced. It has been shown by He [H2] and by Springer (unpublished) that any parabolic character sheaf on $\bar{G}$ appears as a constituent of some perverse cohomology sheaf of $\Gamma_{B_{\Delta}}^{G_{\Delta}}\left(C^{\prime}\right)$ for some $C^{\prime}$ as in 1.3 (with $X^{\prime}=\bar{G}$ ). Hence from 1.3(a) we deduce:

(a) If $K$ is a parabolic character sheaf on $\bar{G}$ then $S S(K) \subset \mu_{G \times G}^{-1}\left(\mathcal{N}^{-}\right)$. 
1.7. In the setup of 1.4 let $\Lambda(D)=\mu_{G \times G}^{-1}\left(\mathcal{N}^{-}\right)$. We want to describe the variety $\Lambda(D)$. For $g \in D$ let $I_{g}$ be the isotropy group at $g$ of the $G \times G$-action on $D$ that is, $I_{g}=\left\{\left(g_{1}, g_{2}\right) \in G \times G ; g_{2}=g^{-1} g_{1} g\right\}$. We have $\operatorname{Lie}\left(I_{g}\right)=\left\{\left(y_{1}, y_{2}\right) \in \mathfrak{g} \times \mathfrak{g} ; y_{2}=\right.$ $\left.\operatorname{Ad}(g)^{-1}\left(y_{1}\right)\right\}$ and the annihilator of $\operatorname{Lie}\left(I_{g}\right)$ in $\mathfrak{g}^{*} \times \mathfrak{g}^{*}$ is $\operatorname{Lie}\left(I_{g}\right)^{\perp}=\left\{\left(z_{1}, z_{2}\right) \in\right.$ $\left.\mathfrak{g}^{*} \times \mathfrak{g}^{*} ; z_{1}+\operatorname{Ad}(g)\left(z_{2}\right)=0\right\}$. This may be identified with the fibre of $T^{*} D$ at $g$. Then

$$
\begin{aligned}
& \Lambda(D)=\left\{\left(g, z_{1}, z_{2}\right) \in D \times \mathfrak{g}^{*} \times \mathfrak{g}^{*} ; z_{1}+\operatorname{Ad}(g)\left(z_{2}\right)=0, z_{1}+z_{2}=0, z_{2} \in \mathcal{N}\right\} \\
& =\{(g, z,-z) ; g \in D, z \in \mathcal{N}, \operatorname{Ad}(g)(z)=z\}=\sqcup_{\mathcal{O}} X_{\mathcal{O}}
\end{aligned}
$$

where $\mathcal{O}$ runs over the (finite) set of $\operatorname{Ad}(G)$-orbits on $\mathcal{N}$ which are normalized by some element of $D$ and $X_{\mathcal{O}}=\{(g, z,-z) ; g \in D, z \in \mathcal{O}, \operatorname{Ad}(g)(z)=z\}$. We pick $\xi \in \mathcal{O}$ and let $\mathcal{Z}^{\prime}=\left\{h \in G^{\prime} ; \operatorname{Ad}(h) \xi=\xi\right\}, \mathcal{Z}=\{h \in G ; \operatorname{Ad}(h) \xi=\xi\}$. Let $\underline{\mathcal{Z}}^{\prime}$ (resp. $\underline{\mathcal{Z}}$ ) be the group of connected components of $\mathcal{Z}^{\prime}$ (resp. $\mathcal{Z}$ ). Then $\underline{\mathcal{Z}}^{\prime}$ is a finite group and $\underline{\mathcal{Z}}$ is a subgroup of $\underline{\mathcal{Z}}^{\prime}$. Let $\underline{\mathcal{Z}}_{1}$ be the set of connected components of $\mathcal{Z}^{\prime}$ that are contained in $D$. Then $\underline{\mathcal{Z}}_{1}$ is a subset of $\mathcal{Z}^{\prime}$; also, $\underline{\mathcal{Z}}$ acts on $\underline{\mathcal{Z}}_{1}$ by conjugation inside $\underline{\mathcal{Z}^{\prime}}$. Let $F_{\mathcal{O}}^{D}$ be the set of orbits of this action. Note that $F_{\mathcal{O}}^{D}$ is independent (up to unique isomorphism) of the choice of $\xi$.

Let $\tilde{X}=\left\{(g, r) \in D \times G ; r^{-1} g r \in \mathcal{Z}^{\prime}\right\}$. Then $\mathcal{Z}$ acts freely on $\tilde{X}$ by $h$ : $(g, r) \mapsto\left(g, r h^{-1}\right)$ and we have an isomorphism $\mathcal{Z} \backslash \tilde{X} \stackrel{\sim}{\longrightarrow} X_{\mathcal{O}},(g, r) \mapsto(g, \operatorname{Ad}(r) \xi)$. By the change of variable $(g, r) \mapsto\left(g^{\prime}, r\right), g^{\prime}=r^{-1} g r, \tilde{X}$ becomes $\left\{\left(g^{\prime}, r\right) ; g^{\prime} \in\right.$ $\left.\mathcal{Z}^{\prime} \cap D, r \in G\right\}$. In the new coordinates, the free action of $\mathcal{Z}$ on $\tilde{X}$ is $h:\left(g^{\prime}, r\right) \mapsto$ $\left(h g^{\prime} h^{-1}, r h^{-1}\right)$. We see that $\tilde{X}$ is smooth of pure $\operatorname{dimension} \operatorname{dim}(\mathcal{Z} \times G)$ and its connected components are indexed naturally by $\underline{\mathcal{Z}}_{1}$ (the connected component containing $\left(g^{\prime}, r\right)$ is indexed by the image of $g^{\prime}$ in $\left.\underline{\mathcal{Z}}_{1}\right)$. The action of $\mathcal{Z}$ on $\tilde{X}$ permutes the connected components of $\tilde{X}$ according to the action of $\underline{\mathcal{Z}}$ on $\underline{\mathcal{Z}}_{1}$ considered above. We see that $X_{\mathcal{O}}=\mathcal{Z} \backslash \tilde{X}$ is smooth of pure $\operatorname{dimension} \operatorname{dim} G$ and its connected components are indexed naturally by the set $F_{\mathcal{O}}^{D}$.

We see that $\Lambda(D)$ can be partitioned into finitely many locally closed, irreducible, smooth subvarieties of $\operatorname{dimension} \operatorname{dim} G$, indexed by the finite set $F(D):=$ $\sqcup_{\mathcal{O}} F_{\mathcal{O}}^{D}$. In particular, $\Lambda(D)$ has pure dimension $\operatorname{dim} G$. More precisely, one checks that

(a) $\Lambda(D)$ is a closed Lagrangian subvariety of $T^{*} D$.

1.8. In the setup of 1.5 we set $\Lambda\left(Z_{J, y, \delta}\right)=\mu_{G \times G}^{-1}\left(\mathcal{N}^{-}\right)$. We want to describe the variety $\Lambda\left(Z_{J, y, \delta}\right)$.

Following [L2, 8.18] we consider the partition $Z_{J, y, \delta}=\sqcup_{\mathbf{s}} Z_{J, y, \delta}^{\mathrm{s}}$ where $Z_{J, y, \delta}^{\mathrm{s}}$ are certain locally closed smooth irreducible $G_{\Delta}$-stable subvarieties of $Z_{J, Y, \delta}$ indexed by the elements $\mathbf{s}$ of a finite set $S(J, \operatorname{Ad}(y) \delta)$ which is in canonical bijection with $J^{\prime} \mathbf{W}$, see $[\mathrm{L} 1,2.5]$. Note that each $\mathbf{s}$ is a sequence $\left(J_{n}, J_{n}^{\prime}, u_{n}\right)_{n \geq 0}$ where $J_{n}, J_{n}^{\prime}$ are subsets of $\mathbf{I}$ such that $J_{n}, J_{n}^{\prime}$ are independent of $n$ for large $n$ and $u_{n} \in \mathbf{W}$ is 1 for large $n$.

We wish to define a Lagrangian subvariety $\Lambda\left(Z_{J, y, \delta}^{\mathbf{s}}\right)$ of $T^{*}\left(Z_{J, y, \delta}^{\mathbf{s}}\right)$. 
Assume first that $\mathbf{s}$ is such that $J_{n}=J, J_{n}^{\prime}=J^{\prime}, u_{n}=1$ for all $n$. In this case we have $J=J^{\prime}$. Let $P \in \mathcal{P}_{J}$ and let $L$ be a Levi subgroup of $P$. Then $\mathbf{d}_{\mathbf{s}}=\left\{g \in D ; g L g^{-1}=L, \operatorname{pos}\left(P, g P g^{-1}\right)=y\right\}$ is a connected component of the algebraic group $N_{G^{\prime}}(L)$ with identity component $L$. Hence $\Lambda\left(\mathbf{d}_{\mathbf{s}}\right) \subset T^{*} \mathbf{d}_{\mathbf{s}}$ is defined as in 1.7. We have a diagram $Z_{J, y, \delta}^{\mathbf{s}} \stackrel{\alpha}{\leftarrow} G \times \mathbf{d}_{\mathbf{s}} \stackrel{p r_{2}}{\longrightarrow} \mathbf{d}_{\mathbf{s}}$ where $\alpha(h, g)=$ $\left(h P h^{-1}, h P h^{-1}, U_{h P h^{-1}} h g h^{-1} U_{h P h^{-1}}\right)$. Note that $\alpha$ is a principal $P$-bundle where $P$ acts on $G \times \mathbf{d}_{\mathbf{s}}$ by $p:(h, g)=\left(h p^{-1}, \bar{p} g \bar{p}^{-1}\right)$ (we denote the canonical homomorphism $P \rightarrow L$ by $\bar{p})$. Let $\Lambda^{\prime}=p r_{2}^{\star} \Lambda\left(\mathbf{d}_{\mathbf{s}}\right) \subset T^{*}\left(G \times \mathbf{d}_{\mathbf{s}}\right)$. By 1.1(a) and 1.7(a), $\Lambda^{\prime}$ is a closed Lagrangian subvariety of $T^{*}\left(G \times \mathbf{d}_{\mathbf{s}}\right)$. It is clearly stable under the natural action of $P$ on $T^{*}\left(G \times \mathbf{d}_{\mathbf{s}}\right)$ (since $\Lambda\left(\mathbf{d}_{\mathbf{s}}\right)$ is $L$-stable). By $1.1(\mathrm{~b})$ there is a unique Lagrangian subvariety $\Lambda^{\prime \prime}$ of $T^{*}\left(Z_{J, y, \delta}^{\mathbf{s}}\right)$ such that $\alpha^{\star} \Lambda^{\prime \prime}=\Lambda^{\prime}$. We set $\Lambda\left(Z_{J, y, \delta}^{\mathbf{s}}\right)=\Lambda^{\prime \prime}$.

We now consider a general $\mathbf{s}=\left(J_{n}, J_{n}^{\prime}, u_{n}\right)_{n \in \mathbf{N}}$. For any $r \in \mathbf{N}$ let $\mathbf{s}_{r}=$ $\left(J_{n}, J_{n}^{\prime}, u_{n}\right)_{n \geq r}, y_{r}=u_{r-1}^{-1} \ldots u_{1}^{-1} u_{0}^{-1} y$. Then $Z_{J_{r}, y_{r}, \delta}^{\mathbf{s}_{r}}$ is defined and we have a canonical map $f_{r}: Z_{J, y, \delta}^{\mathbf{s}} \rightarrow Z_{J_{r}, y_{r}, \delta}^{\mathbf{s}_{r}}$ (a composition of affine space bundles, see $[\mathrm{L} 2,8.20(\mathrm{a})])$. Moreover for sufficiently large $r, \mathbf{s}_{r}, J_{r}, y_{r}, f_{r}$ are independent of $r$; we write $\mathbf{s}_{\infty}, J_{\infty}, y_{\infty}, f_{\infty}$ instead of $\mathbf{s}_{r}, J_{r}, y_{r}, f_{r}$. Note also that $\mathbf{s}_{\infty}, J_{\infty}, y_{\infty}$ are of the type considered earlier, so that $\Lambda\left(Z_{J_{\infty}, y_{\infty}, \delta}^{\mathbf{s}_{\infty}}\right)$ is defined as above. We set $\Lambda\left(Z_{J, y, \delta}^{\mathbf{s}}\right)=f_{\infty}^{\star}\left(\Lambda\left(Z_{J_{\infty}, y_{\infty}, \delta}^{\mathbf{s}_{\infty}}\right)\right)$.

We now define

$$
\Lambda^{\prime}\left(Z_{J, y, \delta}\right)=\sqcup_{\mathbf{s} \in S(J, \operatorname{Ad}(y) \delta)}\left(i_{\mathbf{s}}\right) \star\left(\Lambda\left(Z_{J, y, \delta}^{\mathbf{s}}\right)\right)
$$

where $i_{\mathbf{s}}: Z_{J, y, \delta}^{\mathbf{s}} \rightarrow Z_{J, y, \delta}$ is the inclusion. From $1.1(\mathrm{c})$ we see that $\Lambda^{\prime}\left(Z_{J, y, \delta}\right)$ is a finite union of locally closed Lagrangian subvarieties of $T^{*}\left(Z_{J, y, \delta}\right)$.

We state the following result:

Proposition 1.9. We have $\Lambda\left(Z_{J, y, \delta}\right)=\Lambda^{\prime}\left(Z_{J, y, \delta}\right)$. In particular, $\Lambda^{\prime}\left(Z_{J, y, \delta}\right)$ is closed in $T^{*}\left(Z_{J, y, \delta}\right)$ and $\Lambda\left(Z_{J, y, \delta}\right)$ is a Lagrangian subvariety of $T^{*}\left(Z_{J, y, \delta}\right)$.

Let $x \in Z_{J, y, \delta}^{\mathrm{s}}$. We will show that $\Lambda\left(Z_{J, y, \delta}\right) \cap T_{x}^{*}\left(Z_{J, y, \delta}\right)=\Lambda^{\prime}\left(Z_{J, y, \delta}\right) \cap T_{x}^{*}\left(Z_{J, y, \delta}\right)$.

We identify $\mathfrak{g}$ with $\mathfrak{g}^{*}$ via a $G$-invariant symmetric bilinear form. Choose an element $g_{0}$ in $D$ that normalizes $B$ and a maximal torus $T$ of $B$. Let $P_{J}$ be the unique element in $\mathcal{P}_{J}$ that contains $B$. For $w \in W$, we choose a representative $\dot{w}$ of $w$ in $N(T)$. Set $h_{J, y, \delta}=\left(P_{J},{ }^{\dot{y}^{-1}} P_{J^{\prime}}, U_{\dot{y}-1} P_{J^{\prime}} g_{0} U_{P_{J}}\right) \in Z_{J, y, \delta}$. The element $\mathbf{s} \in S(J, \operatorname{Ad}(y) \delta)$ corresponds to an element $w^{\prime} \in J^{\prime} W$ under the bijection in [L1, 2.5]. Set $w=\left(w^{\prime}\right)^{-1} y \in w \in W^{\delta(J)}$. By [H1, 1.10],

$$
Z_{J, y, \delta}^{\mathbf{s}}=G_{\Delta}\left(P_{J_{\infty}} \dot{w}, 1\right) \cdot h_{J, y, \delta}=G_{\Delta}\left(L_{J_{\infty}} \dot{w}, 1\right) \cdot h_{J, y, \delta}
$$

Note that $\Lambda\left(Z_{J, y, \delta}\right)$ and $\Lambda^{\prime}\left(Z_{J, y, \delta}\right)$ are stable under the action of $G_{\Delta}$. Then we may assume that $x=\left(l_{1} \dot{w}, 1\right) \cdot h_{J, y, \delta}$ for some $l_{1} \in L_{J_{\infty}}$. By $[\mathrm{H} 1,1.10(2)]$, $\left(P_{J_{\infty}}\right)_{\Delta} \cdot\left(L_{J_{\infty}}, 1\right) x=\left(P_{J_{\infty}}, 1\right) \cdot x$, where $\left(P_{J_{\infty}}\right)_{\Delta}=\left\{\left(g, g^{\prime}\right) \in P_{J_{\infty}} \times P_{J_{\infty}} ; g=g^{\prime}\right\}$. For $u \in U_{P_{J_{\infty}}},(u, 1) \cdot x=\left(u^{\prime} l, u^{\prime}\right) \cdot x$ for some $u^{\prime} \in U_{P_{J_{\infty}}}$ and $l \in L_{J_{\infty}}$. Note that 
$\left(U_{P_{J_{\infty}}}, U_{P_{J_{\infty}}}\right) \cdot(l, 1) \cdot x=\left(U_{P_{J_{\infty}}} l, 1\right) \cdot x$ and $\left(P_{J_{\infty}}, 1\right) \cdot x \cong U_{P_{J_{\infty}}} \times\left(L_{J_{\infty}}, 1\right) \cdot x$. Thus $(l, 1) \cdot x=x$ and $\left(U_{P_{J_{\infty}}}, 1\right) \cdot x \subset\left(P_{J_{\infty}}\right)_{\Delta} \cdot x$. Therefore, for $(f,-f) \in$ $\mu_{G \times G}\left(T_{x}^{*}\left(Z_{J, y, \delta}\right)\right)$, we have that $(f,-f)\left(\operatorname{Lie}\left(U_{P_{J_{\infty}}}\right), 0\right)=(f,-f)\left(\operatorname{Lie}\left(P_{J_{\infty}}\right) \Delta\right)=0$, i. e., $f \in \operatorname{Lie}\left(P_{J_{\infty}}\right)$. In particular, $f$ is nilpotent if and only if the image of $f$ under $\operatorname{Lie}\left(P_{J_{\infty}}\right) \rightarrow \operatorname{Lie}\left(P_{J_{\infty}}\right) / \operatorname{Lie}\left(U_{P_{J_{\infty}}}\right) \cong \operatorname{Lie}\left(L_{J_{\infty}}\right)$ is nilpotent.

Hence $\mu_{G \times G}\left(\Lambda\left(Z_{J, y, \delta}\right) \cap T_{x}^{*}\left(Z_{J, y, \delta}\right)\right)$ consists elements of the form $(u+l,-u-l)$ with $u \in \operatorname{Lie}\left(U_{P_{J_{\infty}}}\right), l$ nilpotent in $\operatorname{Lie}\left(L_{J_{\infty}}\right)$ and $(u,-u) I_{x}=(l,-l) I_{x}=0$, where $I_{x}$ is the Lie subalgebra of the isotropic subgroup of $G \times G$ at point $x$.

Denote by $N_{x}$ the stalk at point $x$ of the conormal bundle $N_{Z_{J, y, \delta}^{\mathrm{s}}}^{*}\left(Z_{J, y, \delta}\right)$. Since $Z_{J, y, \delta}^{\mathrm{s}}=G_{\Delta}\left(P_{J_{\infty}}, 1\right) h_{J, y, \delta}$, we have

$$
\mu_{G \times G}\left(N_{x}\right)=\left\{(u,-u) ; u \in \operatorname{Lie}\left(U_{P_{J_{\infty}}}\right),(u,-u) I_{x}=0\right\} .
$$

Let $p_{x}: T_{x}^{*}\left(Z_{J, y, \delta}\right) \rightarrow T_{x}^{*}\left(Z_{J, y, \delta}^{\mathbf{s}}\right) \cong T_{x}^{*}\left(Z_{J, y, \delta}\right) / N_{x}$ be the obvious surjective map. Then $\Lambda\left(Z_{J, y, \delta}\right) \cap T_{x}^{*}\left(Z_{J, y, \delta}\right)=p_{x}^{-1}\left(p_{x}\left(\Lambda\left(Z_{J, y, \delta}\right) \cap T_{x}^{*}\left(Z_{J, y, \delta}\right)\right)\right)$. Note that

$I_{x}=\left\{\left(u_{1}+\operatorname{Ad}\left(l_{1} \dot{w} g_{0}\right) l, u_{2}+l\right) ; u_{1} \in \operatorname{Ad}\left(l_{1} \dot{w} \dot{y}^{-1}\right) \operatorname{Lie}\left(U_{P_{J^{\prime}}}\right), u_{2} \in \operatorname{Lie}\left(U_{P_{J}}\right), l \in \operatorname{Lie}\left(L_{J}\right)\right\}$.

Thus for $l \in \operatorname{Lie}\left(L_{J_{\infty}}\right),(l,-l) I_{x}=0$ if and only if $\operatorname{Ad}\left(l_{1} \dot{w} g_{0}\right) l=l$.

We identify $T_{x}^{*}\left(Z_{J, y, \delta}\right)$ with $\mu_{G \times G}\left(T_{x}^{*}\left(Z_{J, y, \delta}\right)\right) \subset \mathfrak{g} \times \mathfrak{g}$ and regard $T_{x}^{*}\left(Z_{J, y, \delta}^{\mathbf{s}}\right)$ as a subspace of $(\mathfrak{g} \times \mathfrak{g}) / N_{x}$. Set $M=\left\{(l,-l) ; l \in \operatorname{Lie}\left(L_{J_{\infty}}\right), \operatorname{Ad}\left(l_{1} \dot{w} g_{0}\right) l=l\right\} \subset \mathfrak{g} \times \mathfrak{g}$. Then

(1) $p_{x}\left(\Lambda\left(Z_{J, y, \delta}\right) \cap T_{x}^{*}\left(Z_{J, y, \delta}\right)\right)=\left(M+N_{x}\right) / N_{x}$.

Now consider the commuting diagram

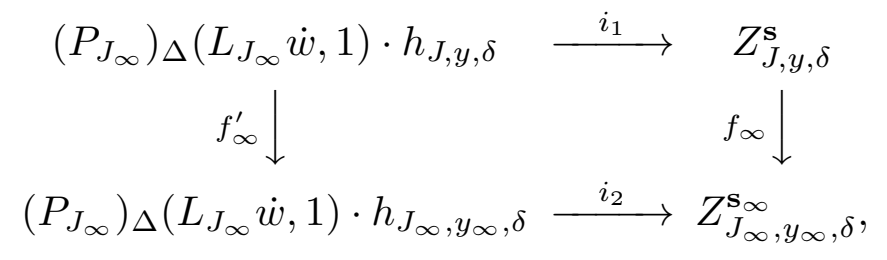

where $i_{1}, i_{2}$ are inclusions and $f_{\infty}^{\prime}$ is the restriction of $f_{\infty}$. Let $x^{\prime}=f_{\infty}(x) \in$ $Z_{J_{\infty}, y_{\infty}, \delta}^{\mathbf{s}}$. Since $f_{\infty}^{\prime}$ is $P_{J_{\infty}} \times P_{J_{\infty}}$-invariant, we have the following commuting diagram

$$
\begin{aligned}
T_{x^{\prime}}^{*}\left(\left(P_{J_{\infty}}\right)_{\Delta}\left(L_{J_{\infty}} \dot{w}, 1\right) \cdot h_{J_{\infty}, y_{\infty}, \delta}\right) & \stackrel{{ }^{1} \mu_{P_{J_{\infty}} \times P_{J_{\infty}}}}{\longrightarrow} \operatorname{Lie}\left(P_{J_{\infty}}\right)^{*} \times \operatorname{Lie}\left(P_{J_{\infty}}\right)^{*} \\
\left(f_{\infty}^{\prime}\right)^{*} \downarrow & \\
T_{x}^{*}\left(\left(P_{J_{\infty}}\right)_{\Delta}\left(L_{J_{\infty}} \dot{w}, 1\right) \cdot h_{J, y, \delta}\right) & \stackrel{{ }^{2} \mu_{P_{J_{\infty}} \times P_{J_{\infty}}}}{\longrightarrow} \operatorname{Lie}\left(P_{J_{\infty}}\right)^{*} \times \operatorname{Lie}\left(P_{J_{\infty}}\right)^{*},
\end{aligned}
$$

where ${ }^{1} \mu_{P_{J_{\infty}} \times P_{J_{\infty}}}$ and ${ }^{2} \mu_{P_{J_{\infty}} \times P_{J_{\infty}}}$ are the moment maps. Since the actions of $P_{J_{\infty}} \times P_{J_{\infty}}$ on $\left(P_{J_{\infty}}\right)_{\Delta}\left(L_{J_{\infty}} \dot{w}, 1\right) \cdot h_{J, y, \delta}$ and $\left(P_{J_{\infty}}\right)_{\Delta}\left(L_{J_{\infty}} \dot{w}, 1\right) \cdot h_{J_{\infty}, y_{\infty}, \delta}$ are transitive, ${ }^{1} \mu_{P_{J_{\infty}} \times P_{J_{\infty}}}$ and ${ }^{2} \mu_{P_{J_{\infty}} \times P_{J_{\infty}}}$ are injective. 


$$
\begin{aligned}
& \text { Set } \Lambda_{x^{\prime}}\left(Z_{J_{\infty}, y_{\infty}, \delta}^{\mathbf{s}_{\infty}}\right)=\Lambda\left(Z_{J_{\infty}, y_{\infty}, \delta}^{\mathbf{s}_{\infty}}\right) \cap T_{x^{\prime}}^{*}\left(Z_{J_{\infty}, y_{\infty}, \delta}^{\mathbf{s}_{\infty}}\right) \text {. Then } \\
& { }^{2} \mu_{P_{J_{\infty}} \times P_{J_{\infty}}}\left(i_{2}^{*}\left(\Lambda_{x^{\prime}}\left(Z_{J_{\infty}, y_{\infty}, \delta}^{\mathbf{s}_{\infty}}\right)\right)={ }^{1} \mu_{P_{J_{\infty}} \times P_{J_{\infty}}}\left(\left(f_{\infty}^{\prime}\right)^{*} i_{2}^{*}\left(\Lambda_{x^{\prime}}\left(Z_{J_{\infty}, y_{\infty}, \delta}^{\mathbf{s}_{\infty}}\right)\right)=M .\right.\right.
\end{aligned}
$$

Here we identify $\operatorname{Lie}\left(P_{J_{\infty}}\right)^{*}$ with $\operatorname{Lie}\left(P_{J_{\infty}}^{-}\right)$via the symmetric bilinear form. Moreover, $i_{1}^{*} f_{\infty}^{*}=\left(f_{\infty}^{\prime}\right)^{*} i_{2}^{*}$ maps $\Lambda_{x^{\prime}}\left(Z_{J_{\infty}, y_{\infty}, \delta}^{\mathbf{s}_{\infty}}\right)$ bijectively onto its image. Therefore ${ }^{1} \mu_{P_{J_{\infty}} \times P_{J_{\infty}}}\left(i_{1}^{*}\left(\Lambda\left(Z_{J, y, \delta}^{\mathbf{s}}\right) \cap T_{x}^{*}\left(Z_{J, y, \delta}^{\mathbf{s}}\right)\right)\right)=M$ and $i_{1}^{*} \operatorname{maps} \Lambda\left(Z_{J, y, \delta}^{\mathbf{s}}\right) \cap T_{x}^{*}\left(Z_{J, y, \delta}^{\mathbf{s}}\right)$ bijectively onto its image. In other words,

(2) $\Lambda\left(Z_{J, y, \delta}^{\mathbf{s}}\right) \cap T_{x}^{*}\left(Z_{J, y, \delta}^{\mathbf{s}}\right)=\left(M+N_{x}\right) / N_{x}$.

Combining (1) and $(2), p_{x}\left(\Lambda\left(Z_{J, y, \delta}\right) \cap T_{x}^{*}\left(Z_{J, y, \delta}\right)\right)=\Lambda\left(Z_{J, y, \delta}^{\mathbf{s}}\right) \cap T_{x}^{*}\left(Z_{J, y, \delta}^{\mathbf{s}}\right)$. The proposition is proved.

Corollary 1.10. The set of irreducible components of $\Lambda\left(Z_{J, y, \delta}\right)$ is in natural bijection with $\sqcup_{\mathbf{s} \in S(J, \operatorname{Ad}(y) \delta)} F\left(\mathbf{d}_{\mathbf{s}_{\infty}}\right)$ (notation of 1.7, 1.8).

1.11. In the setup of 1.6 let $\Lambda(\bar{G})=\mu_{G \times G}^{-1}\left(\mathcal{N}^{-}\right)$. We want to describe the variety $\Lambda\left(Z_{J, y, \delta}\right)$. As in $[\mathrm{L} 2,12.3]$, we have $\bar{G}=\sqcup_{J \subset \mathbf{I}} G_{J}$ where $G_{J}$ are the various $G \times G$ orbits in $\bar{G}$; moreover we may identify $G_{J}=T_{J} \backslash Z_{J, y_{J}, 1}$ where $y_{J}$ is the longest element in $\mathbf{W}^{J}$ and $T_{J}$ is a torus acting freely on $Z_{J, y_{J}, 1}$. Let $a_{J}: Z_{J, y_{J}, 1} \rightarrow G_{J}$ be the canonical map.

For each $J$ let $\mu_{G \times G ; J}: G_{J} \rightarrow \mathfrak{g}^{*} \times \mathfrak{g}^{*}$ be the moment map of the restriction of the $G \times G$-action on $\bar{G}$ to $G_{J}$. Let $i_{J}: G_{J} \rightarrow \bar{G}$ be the inclusion. From the definitions, we have

(a) $\Lambda(\bar{G})=\sqcup_{J \subset \mathbf{I}}\left(i_{J}\right)_{\star} \mu_{G \times G ; J}^{-1}\left(\mathcal{N}^{-}\right)$

and $\Lambda\left(Z_{J, y_{J}, 1}\right)=a_{J}^{\star}\left(\mu_{G \times G ; J}^{-1}\left(\mathcal{N}^{-}\right)\right)$.

Since $\Lambda\left(Z_{J, y_{J}, 1}\right)$ is a $T_{J}$-stable Lagrangian subvariety of $T^{*}\left(Z_{J, y_{J}, 1}\right)$ (see 1.9$)$, it follows that $\mu_{G \times G ; J}^{-1}\left(\mathcal{N}^{-}\right)$is a Lagrangian subvariety of $T^{*}\left(G_{J}\right)$. Hence, using $1.1(\mathrm{c})$, we see that $\left(i_{J}\right)_{\star} \mu_{G \times G ; J}^{-1}\left(\mathcal{N}^{-}\right)$is a Lagrangian subvariety of $T^{*} \bar{G}$. Using this and (a) we see that

(b) $\Lambda(\bar{G})$ is a Lagrangian subvariety of $T^{*} \bar{G}$.

From the previous proof we see that

(c) The set of irreducible components of $\Lambda(\bar{G})$ is in natural bijection with $\sqcup_{J \subset \mathbf{I}} \sqcup_{\mathbf{s} \in S\left(J, \operatorname{Ad}\left(y_{J}\right)\right)} F\left(\mathbf{d}_{\mathbf{s}_{\infty}}\right)$ (notation of 1.7, 1.8).

1.12. Let $X=Z_{J, y, \delta}$ or $\bar{G}$. There is a well-defined map from the irreducible components of $\Lambda(X)$ to the nilpotent conjugacy classes of $\mathfrak{g}^{*}$ which sends the irreducible component $C$ of $\Lambda(X)$ to the nilpotent conjugacy class $\mathcal{O}$, where $\mu_{G \times G}(C) \cap\{(f,-f) \in \mathcal{O} \times \mathcal{O}\}$ is dense in $\mu_{G \times G}(C)$.

\section{REFERENCES}

[Gi] V. Ginzburg, Admissible modules on a symmetric space, Astérisque 173-174 (1989), 199256.

[H1] X. He, The G-stable pieces of the wonderful compactification, math.RT/0412302.

[H2] X. He, The character sheaves on the group compactification, math.RT/0508068. 
[KS] M. Kashiwara and P. Schapira, Sheaves on manifolds, Springer-Verlag, Berlin, 1994.

[L1] G. Lusztig, Parabolic character sheaves I, Moscow Math.J 4 (2004), 153-179.

[L2] G. Lusztig, Parabolic character sheaves II, Moscow Math.J. 4 (2004), 869-896.

[MV] I. Mirković and K. Vilonen, Characteristic varieties of character sheaves, Invent.Math. 93 (1988), 405-418.

[S] T. A. Springer, Intersection cohomology of $B \times B$-orbit closures in group compactifications, J.Alg. 258 (2002), 71-111.

School of Mathematics, Institute for Advanced Study, Princeton, NJ 08540

E-mail address: hugo@math.ias.edu

Department of Mathematics, M.I.T., Cambridge, MA 02139

E-mail address: gyuri@math.mit.edu 